\title{
Therapeutic effect of Resveratrol in the treatment of osteoarthritis via the MALAT1/miR-9/NF-кB signaling pathway
}

\author{
GUOPENG ZHANG ${ }^{1}$, HUA ZHANG ${ }^{1}$, WULIN YOU ${ }^{2}$, XIAOCHEN TANG ${ }^{3},{\text { XIUFANG } \text { LI }^{4} \text { and ZHENGFENG GONG }}^{1}$ \\ ${ }^{1}$ Department of Orthopedics and Traumatology, Nanjing University of Chinese Medicine, Nanjing, Jiangsu 210023; \\ ${ }^{2}$ Department of Orthopedics and Traumatology, Wuxi Hospital of Chinese Medicine, Wuxi, Jiangsu 214001; \\ ${ }^{3}$ Department of Orthopedics and Traumatology, Suzhou Hospital of Chinese Medicine, Suzhou, Jiangsu 215009; \\ ${ }^{4}$ Department of Science and Education, Xuyi People's Hospital, Huaian, Jiangsu 211700, P.R. China
}

Received April 29, 2018; Accepted December 21, 2018

DOI: $10.3892 /$ etm.2020.8471

\begin{abstract}
The aim of the current study was to explore the role of Resveratrol (Res) in osteoarthritis (OA) and its underlying mechanism. Reverse transcription-quantitative polymerase chain reaction and western blot analysis were used to determine the relative expression levels of metastasis-associated lung adenocarcinoma transcript 1 (MALAT1), microRNA-9 (miR-9), nuclear factor kappa B subunit 1 (NF-kB1), interleukin (IL)-6, matrix metallopeptidase 13 (MMP-13) and caspase- 3 in vitro and in the in vivo model of OA, as well as examining the effect of Res on MALAT1, miR-9 and NF- $\kappa$ B1, IL-6, MMP-13 and caspase-3 expression levels. Immunohistochemical analysis was performed to examine NF- $\mathrm{kB} 1$ and MMP-13 protein levels in the in vivo model of OA. Dual-luciferase reporter assays were used to confirm the regulatory relationship between miR-9 and MALAT1 and NF-kB1, as well as examining the effect of Res on the transcriptional activation of MALAT1 promoter. Furthermore, the effect of Res on cell proliferation in vitro was examined by MTT assay. The relative mRNA expression levels of MALAT1 and NF- $\mathrm{BB} 1$ were significantly increased, while miR-9 expression was significantly decreased in the OA group compared with the sham group. Treatment with Res partially reversed the effects of OA on MALAT1, NF-kB1 and miR-9 expression. Similarly, the relative protein expression levels of NF- $\kappa B 1$, IL-6, MMP-13 and caspase-3 were significantly increased in the OA group compared with the sham group; however, treatment with Res partially reversed the effects of OA on the protein expression levels of NF- $\mathrm{KB} 1, \mathrm{IL}-6, \mathrm{MMP}-13$ and caspase-3. MALAT1 and NF- $\mathrm{kB} 1$ were identified as potential target genes of miR-9, and dual-luciferase assays were used to
\end{abstract}

Correspondence to: Professor Zhengfeng Gong, Department of Orthopedics and Traumatology, Nanjing University of Chinese Medicine, 282 Hanzhong Road, Nanjing, Jiangsu 210023, P.R. China E-mail: osteo_joint@yeah.net

Key words: osteoarthritis, chondrocytes, resveratrol, metastasis associated lung adenocarcinoma transcript 1, miR-9, nuclear factor kappa B subunit 1, apoptosis examine the effect of miR-9 on the luciferase activity of 3'UTR MALAT1 and NF- $\kappa B 1$. Treatment with Res suppressed the transcriptional activation of the MALAT1 promoter, thereby inhibiting MALAT1 expression. Additionally, the relative expression level of miR-9 significantly increased following treatment with Res in a dose-dependent manner, while the relative protein expression levels of NF-кB1, IL-6, MMP-13 and caspase- 3 significantly decreased following treatment with Res compared with the control. Furthermore, treatment with Res significantly increased the growth rate of chondrocytes in a dose-dependent manner compared with the control. Taken together, these results suggest that direct targeting of the MALAT1/miR-9/NF-кB1/IL-6, MMP-13/caspase-3 axis may be a novel therapeutic strategy for the treatment of OA.

\section{Introduction}

Osteoarthritis (OA) is the most common form of rheumatic disease with the highest rate of incidence, which ultimately leads to limited joint mobility, chronic pain and disability (1). At the cellular level, OA is characterized by decreased tissue cellularity and damage to the extracellular matrix (1). It was previously demonstrated that apoptosis in chondrocytes was enhanced in the articular cartilage of patients with OA. Apoptotic cells in the cartilage of patients with OA were identified by the detection of DNA strand breaks using TUNEL assay, which identified high levels of apoptotic cells in the zones of cartilage known as the superficial and middle zones (2).

Resveratrol (Res) can regulate the expression of several intracellular signaling proteins and it is known to be associated with specific anti-inflammatory properties $(3,4)$. In addition, Res can regulate cell proliferation while preventing inflammation and apoptosis in both the chronic and acute phases of OA (5). Res reduces the morphological changes of chondrocytes and inhibits the induction of the pro-inflammatory cytokine interleukin (IL)-1 $\beta$ (4). Furthermore, Res suppresses nuclear factor kappa B subunit 1 (NF- $\kappa$ B1)-dependent pro-inflammatory signaling and inhibits membrane-bound and mature IL-1 $\beta$ production in chondrocytes (3). By contrast, in vitro studies demonstrated that IL- $1 \beta$ inhibits the chondrocyte proliferation (3-5). 
Until recently, non-coding RNAs (ncRNAs) were considered to have generic intracellular roles (6). Ribosomal RNAs (rRNAs) and transfer RNAs (tRNAs) are involved in the translation of mRNA, whereas small nuclear RNAs (snRNAs) participate in RNA splicing and small nucleolar RNAs (snoRNAs) mediate rRNA modification (6). Previous studies demonstrated that long non-coding RNAs (lncRNAs), RNAs $>200$ nucleotides in length with no or limited protein-coding ability $(6,7)$, can serve crucial roles in several types of human cancer (8-10). In addition, lncRNAs may function to regulate gene expression at both the transcriptional and post-transcriptional levels based on genetic and epigenetic mechanisms $(11,12)$. Furthermore, associations between lncRNAs and OA were previously investigated. Xing et al (13) identified 121 lncRNAs that were up- or downregulated in OA. MicroRNAs (miR) are small non-coding RNA molecules derived from the introns and exons of both protein-coding and non-coding transcripts transcribed by RNA polymerase II (13-15). In addition, processed pseudogenes can activate certain miRs (16).

A previous study demonstrated that treatment with Res downregulated the expression of MALAT1, and as an lncRNA, MALAT1 can function as a molecular sponge of miR-9 (17). In addition, miR-9 can directly target NF- $\mathrm{B}$, and as an inflammatory cytokine $\mathrm{NF}-\kappa \mathrm{B}$ can induce apoptosis in chondrocytes contributing to the development of OA (18). In the current study, to explore the role of Res in OA and its underlying mechanism, the in vivo model of OA was established and the effect of Res was examined in vitro and in the in vivo model of OA. PCR techniques and western blot analysis, immunohistochemical analysis, dual-luciferase reporter assays were performed to study underlying mechanisms, while MTT assay was used to study the effect of Res on cell proliferation in vitro. Res treatment was indicated to inhibit MALAT1 and modulate MALAT1/miR-9/NF- $\kappa$ B signaling pathway.

\section{Materials and methods}

Animals and experimental design. A total of 30 male C57BL/6 mice (age, 10 weeks; weight, 20-30 g) were purchased from the Shanghai Laboratory Animal Centre, Chinese Academy of Sciences (Shanghai, China). The mice were divided into three groups: Sham surgery (sham group, $n=10$ ), OA with vehicle injection (OA group, $n=10$ ) and OA with Res treatment $(\mathrm{OA}+\mathrm{Res}$ group, $\mathrm{n}=10)$. Following 7 days acclimatization, OA was induced using the destabilizing medial meniscus (DMM) model (18). Briefly, mice were anesthetized with pentobarbital (50 mg/ $\mathrm{kg}$ IP) and an incision was made in the right knee. The joint capsule immediately medial to the patellar tendon was incised and the joint capsule was opened using microsurgical scissors. DMM was achieved by sectioning the medial meniscotibial ligament with microsurgical scissors. In the sham group, an incision was made in the right knee joint and ligaments were visualized only and not transected. All experimental protocols were approved by the Institutional Animal Care and Use Committee at Nanjing University of Chinese Medicine (Nanjing, China). The current study was approved by the Institutional Ethics Committee on Animal Research at Nanjing University of Chinese Medicine.
Res treatment. Res (Sigma-Aldrich; Merck KGaA, Darmstadt, Germany) was dissolved in dimethyl sulfoxide (DMSO; Sigma-Aldrich; Merck KGaA) to give a final concentration of $100 \mathrm{mg} / \mathrm{ml}$ (stock solution). The stock solution was diluted with PBS to give a final concentration of $12.5 \mathrm{mg} / \mathrm{ml}$. At five weeks post-surgery, mice in the $\mathrm{OA}+$ Res group received $10 \mathrm{ml}$ Res, while mice in the sham group received $10 \mathrm{ml}$ PBS via intra-articular injection through the patellar tendon with the use of a U-100 insulin syringe (BD Biosciences, San Jose, CA, USA). All injections were performed twice a week for 8 weeks. At 13 weeks post-surgery, the mice were sacrificed and knee joints were harvested.

Cell culture and transfection. Mouse chondrocytes (ATCC ${ }^{\circledR}$ CRL-12424 ${ }^{\mathrm{TM}}$ ) were purchased from the American Type Culture Collection (ATCC, Manassas, VA, USA). Chondrocytes were cultured in Dulbecco's modified Eagle medium (DMEM)/F12 (Gibco; Thermo Fisher Scientific, Inc.) supplemented with $10 \% \mathrm{FBS}, 0.1 \mathrm{mg} / \mathrm{ml}$ streptomycin and $1,000 \mathrm{U} / \mathrm{ml}$ penicillin (Thermo Fisher Scientific, Inc.) and maintained in a $5 \% \mathrm{CO}_{2}$-humidified incubator at room temperature. Chondrocytes were grown to $70 \%$ confluence and treated with 15 or $30 \mu \mathrm{M}$ Res prior to transfection with miR-9 mimic or scramble control which were manufactured by Shanghai GenePharma Co., Ltd. using Lipofectamine ${ }^{\circledR}$ 2000 (Invitrogen; Thermo Fisher Scientific, Inc.). Each experiment was performed in triplicate.

RNA isolation and reverse transcription-quantitative polymerase chain reaction ( $R T-q P C R)$. Total RNA was extracted from tissue samples and chondrocytes using the mirVana ${ }^{\mathrm{TM}}$ miRNA Isolation kit (Ambion; Thermo Fisher Scientific, Inc.), according to the manufacturer's protocol. Total RNA was reverse transcribed into cDNA using the PrimeScript ${ }^{\circledR}$ RT reagent kit (Takara Biotechnology Co., Ltd., Dalian, China), followed by qPCR. For miR-9 and MALAT1 expression, qPCR was performed using the TaqMan microRNA assay (Applied Biosystems; Thermo Fisher Scientific, Inc.). For NF- $\kappa \mathrm{B} 1$ expression, qPCR was performed using the SYBR ${ }^{\circledR}$ Premix Ex Taq ${ }^{\mathrm{TM}}$ kit (Takara Biotechnology Co., Ltd.). All qPCR were performed using an ABI-7500 Sequence Detection System (Applied Biosystems; Thermo Fisher Scientific, Inc.). The relative miR-9, MALAT1 and $N F-\kappa B 1$ expression levels were quantified using the $2^{-\Delta \Delta C q}$ method (19). U6 and GAPDH mRNA were used as endogenous controls for miR-9, MALAT1 and NF- $\kappa \mathrm{B} 1$, respectively. The primer pairs used were as follows: miR-9 forward, 5'-GGTCTTTGGTTATCTAGCTGTATGA-3' and reverse, 5'-3CAGTGCGTGTCGTGGAGT-3); MALAT1 forward, 5'-CAGACCACCACAGGTTTACAG-3' and reverse, 5'-AGA CCATCCCAAAATGCTTCA-3'); NF- $\kappa$ B1 forward, 5'-CAA GCGAGGAGGGGACGTG-3' and reverse, 5'-CCCCCA GAGCCTCCACCC-3'); U6 forward, 5'-CTCGCTTCGGCA GCACA-3' and reverse, 5'-AACGCTTCACGAATTTGC GT-3'; GAPDH mRNA forward, 5'-TGACTTCAACAGCGA CACCCA-3' and reverse, 5'-CACCCTGTTGCTGTAGCC AAA-3'. The thermocycling conditions were as follows: $94^{\circ} \mathrm{C}$ for $5 \mathrm{~min}, 30$ cycles of $94^{\circ} \mathrm{C}$ for $30 \mathrm{sec}$ and $60^{\circ} \mathrm{C}$ for $30 \mathrm{sec}$ and $72^{\circ} \mathrm{C}$ for $30 \mathrm{sec}, 72^{\circ} \mathrm{C}$ for $10 \mathrm{~min}$. Each experiment was performed in triplicate. 
MTT assay. Cell proliferation was examined in chondrocytes by MTT assay following treatment with Res. Following a $48 \mathrm{~h}$ incubation, $0.5 \mathrm{mg} / \mathrm{ml}$ MTT (Sigma-Aldrich; Merck $\mathrm{KGaA}$ ) was added to chondrocytes and incubated at $37^{\circ} \mathrm{C}$ for $4 \mathrm{~h}$. Following incubation, culture medium was removed and $150 \mu 1$ DMSO was added into each well (48-well plate at a density of $1 \times 10^{5}$ cells/well). The absorbance was measured at a wavelength of $490 \mathrm{~nm}$ using a multi-mode microplate reader (CHAMELEON $^{\text {тм }} \mathrm{V}$; Hidex, Turku, Finland) to analyze cell survival. All experiments were performed in triplicate.

Dual-luciferase reporter assay. The mutant 3'UTR of MALAT1/NF- $\mathrm{B} 1$ was generated by mutating the miR-9 binding site sequence in the wild-type 3'UTR of MALAT1/NF- $\kappa$ B1. The wild-type or mutant 3'UTR of MALAT1/NF- $\kappa$ B1 were PCR amplified and cloned into the pRL-TK reporter vector (Promega Corporation, Madison, WI, USA). Chondrocytes were seeded into 48-well plates at a density of $1 \times 10^{5}$ cells $/ \mathrm{ml}$ and co-transfected with $300 \mathrm{ng}$ luciferase reporter vector containing the wild-type or mutant 3'UTR of MALAT1/NF- $\mathrm{BB} 1$ and 20 pmol miR-9 mimic or scramble control using Lipofectamine ${ }^{\circledR} 2000$ (Invitrogen; Thermo Fisher Scientific, Inc.), according to the manufacturer's protocol. Following 48-h transfection, chondrocytes were lysed and cell lysates were collected. Relative luciferase activities were detected using a Dual-Luciferase Reporter Assay system (Promega Corporation). Firefly luciferase activity was normalized to Renilla luciferase activity. Each test was performed in triplicate.

MALAT1 luciferase assay. The promoter region of wild-type MALAT1 was PCR amplified and cloned into the pRL-TK reporter vector (Promega Corporation). Chondrocytes were seeded into 48 -well plates at a density of $1 \times 10^{5}$ cells $/ \mathrm{ml}$ and transfected with $300 \mathrm{ng}$ luciferase reporter vector containing the promoter region of MALAT1 using Lipofectamine ${ }^{\circledR}$ 2000 (Invitrogen; Thermo Fisher Scientific, Inc.), according to the manufacturer's protocol. Subsequently, chondrocytes were treated with 15 or $30 \mu \mathrm{M}$ Res for $48 \mathrm{~h}$. Following 48-h treatment with Res, chondrocytes were lysed and cell lysates were collected. The relative Renilla luciferase activity was detected using a Luciferase Reporter Assay system (Promega Corporation). Each experiment was performed in triplicate.

Western blot analysis. Chondrocytes were washed three times with ice-cold PBS and total protein was extracted using $0.2 \mathrm{ml}$ RIPA lysis buffer (Beyotime Institute of Biotechnology, Haimen, China). Chondrocytes were incubated in lysis buffer for $30 \mathrm{~min}$ on ice followed by centrifugation at $18,894 \mathrm{x} g$ for $20 \mathrm{~min}$ at $4^{\circ} \mathrm{C}$. Total protein was quantified using a bicinchoninic acid assay kit (Thermo Fisher Scientific, Inc.) and $25 \mu \mathrm{g}$ protein was separated via SDS-PAGE on a 6 or $10 \%$ gel. The separated proteins were transferred onto polyvinylidene difluoride membranes (EMD Millipore, Billerica, MA) and blocked for $1 \mathrm{~h}$ at $25^{\circ} \mathrm{C}$ with Tris-buffered saline containing $0.1 \%$ Tween $^{\mathrm{TM}} 20$ and $5 \%$ bovine serum albumin (Sigma-Aldrich; Merck KGaA) to prevent non-specific binding. The membranes were incubated with mouse primary antibodies against NF- $\mathrm{B} 1$ (1:5,000; cat. no. MA5-15128), MMP-13 (1:5,000; cat. no. MA5-14247), caspase-3 (1:5,000; cat. no. MA1-91637), IL-6 (1:5,000; cat. no. M621B all Invitrogen; Thermo Fisher Scientific, Inc.) or $\beta$-actin (1:10,000; cat. no. 3700S; Cell Signaling Technology, Inc., Danvers, MA, US) for $12 \mathrm{~h}$ at $4^{\circ} \mathrm{C}$. Following primary incubation, membranes were incubated with anti-mouse horseradish peroxidase (HRP)-labeled secondary antibodies (1:12,000; cat. no. 7076S; Cell Signaling Technology, Inc.) at room temperature for $1 \mathrm{~h}$. Protein bands were visualized using RapidStep ${ }^{\mathrm{TM}} \mathrm{ECL}$ detection reagent (EMD Millipore) and Syngene GeneGenius Gel Light Imaging system (Syngene, Frederick, MD, USA), according to the manufacturer's protocol. Each experiment was performed in triplicate.

Immunohistochemistry (IHC). Tissue samples were fixed with $10 \%$ formalin at $4^{\circ} \mathrm{C}$ for $12 \mathrm{~h}$ and embedded in paraffin and paraffin-embedded samples were cut into 5-mm sections, which was later put to blocking stage with $3 \%$ hydrogen peroxide for $60 \mathrm{~min}$ at room temperature. For antigen retrieval, tissue sections were incubated with $0.01 \mathrm{M}$ sodium citrate (pH 6) in a microwave oven for $10 \mathrm{~min}$. Following antigen retrieval, tissue sections were incubated with mouse primary antibodies against NF-кB1 (cat. no. PA5-17654; 1:500) and MMP-13 (cat. no. MA5-14238; 1:500; both Invitrogen; Thermo Fisher Scientific, Inc.) for $12 \mathrm{~h}$ at $4^{\circ} \mathrm{C}$. Following primary incubation, tissue sections were incubated with HRP-labeled secondary antibodies (cat. no. 7074S; 1:1,000; CST, Danvers, MA, US) for $1 \mathrm{~h}$ at room temperature. Subsequently, tissue sections were stained with hematoxylin (Dako Cytomation, Glostrup, Denmark) at $37^{\circ} \mathrm{C}$ for $2 \mathrm{~h}$. Dimethyl benzene was used to mount the tissue sections and images were captured using a light microscope (magnification, $\mathrm{x} 400$ ). Two independent pathologists scored the staining intensity of each protein. The staining intensity was scored as follows: no staining, 0; weak staining, 1; moderate staining, 2; and strong staining, 3 . Each experiment was performed in triplicate.

Statistical analysis. Data were presented as the mean \pm standard deviation. All statistical analyses were performed using SPSS software (version 16.0; SPSS, Inc., Chicago, IL, USA). The difference between two groups was analyzed using a two-tailed Student's t-test, whilst the difference among three or more groups was analyzed using one-way analysis of variance and Scheffe's test was used as a post-hoc test. $\mathrm{P}<0.05$ was considered to indicate a statistically significant difference.

\section{Results}

MALAT1, miR-9 and NF- $\mathrm{KB} 1$ expression in the in vivo model of $O A$. As shown in Fig. 1, the relative expression levels of MALAT1, miR-9 and NF- $\mathrm{BB} 1$ were analyzed in tissue samples from mice in the sham, $\mathrm{OA}$ and $\mathrm{OA}+$ Res groups. The relative mRNA expression levels of MALAT1 and NF- $\kappa$ B1 were significantly increased, while miR-9 expression was significantly decreased in the OA group compared with the sham group (Fig. 1B-D). Meanwhile, compared with OA group, treatment with Res partially reversed the effects of OA on the mRNA expression levels of MALAT1, miR-9 and NF- $\kappa$ B1.

$N F-\kappa B 1, I L-6, M M P-13$ and caspase-3 expression in the in vivo model of $O A$. The relative protein expression levels of NF-кB1, IL-6, MMP-13 and caspase-3 were determined by 

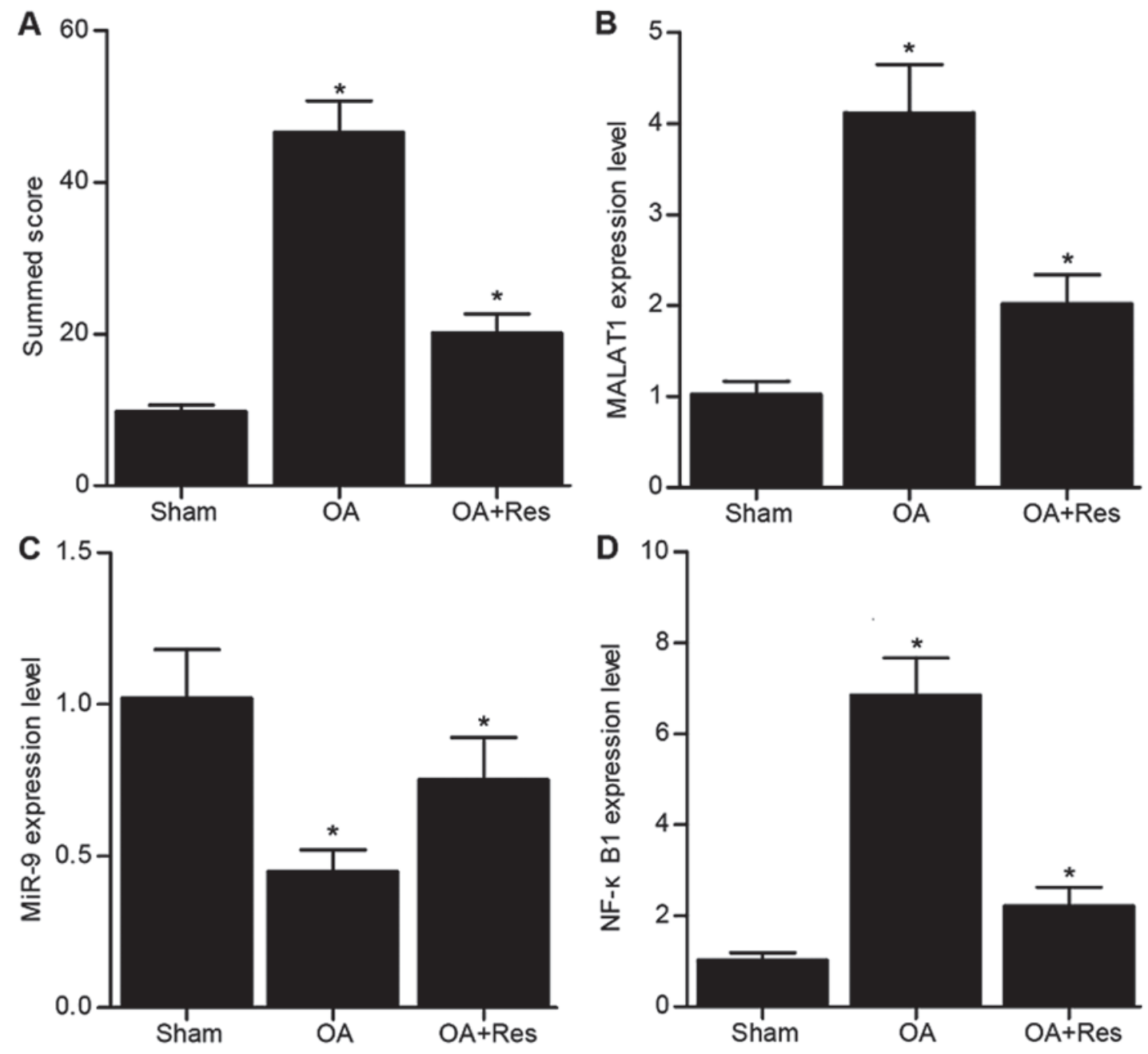

Figure 1. MALAT1, miR-9 and NF-kB1 expression in the in vivo model of OA. The (A) relative (B) MALAT1, (C) miR-9 and (D) NF- $\mathrm{kB} 1$ expression levels were determined by RT-qPCR in tissue samples from mice in the Sham, OA and OA + Res groups. ${ }^{*} \mathrm{P}<0.05$ vs. OA + Res group; ${ }^{* *} \mathrm{P}<0.05$ vs. sham group. MALAT1, metastasis associated lung adenocarcinoma transcript 1; miR, microRNA; NF-кB1, nuclear factor kappa B subunit 1; OA, osteoarthritis; Res, Resveratrol; RT-qPCR, reverse transcription-quantitative polymerase chain reaction.

western blot analysis in tissue samples from mice in the sham, $\mathrm{OA}$ and $\mathrm{OA}+$ Res groups (Fig. 2). The NF-kB1, IL-6, MMP-13 and caspase-3 protein expression levels were significantly increased in the OA group compared with the sham group (Fig. 2B-E). However, treatment with Res partially reversed the effects of OA on the protein expression levels of NF- $\mathrm{KB} 1$, IL-6, MMP-13 and caspase-3.

Immunohistochemical analysis of $N F-\kappa B 1$ and $M M P-13$ in the in vivo model of $O A$. IHC was performed to examine the protein levels of NF- $\mathrm{kB} 1$ and MMP-13 in tissue samples from mice in the sham, OA and OA + Res groups. As shown in Fig. 3, strong NF- $\mathrm{KB} 1$ staining was observed in the OA group compared with the sham group. Similarly, strong MMP-13 staining was observed in the OA group compared with the sham group (Fig. 4). These results suggest that the in vivo DMM-induced OA model increased NF- $\kappa \mathrm{B} 1$ and MMP-13 expression. However, treatment with Res partially reversed the effects of OA on NF-kB1 and MMP-13 expression.

MALAT1 directly regulates miR-9 and miR-9 directly targets $N F-\kappa B 1$. The 3'UTR of MALAT1 was revealed to contain a putative binding site for miR-9 (Fig. 5A) via computational analysis using the online microRNA database (www.mirdb. org). The dual-luciferase reporter gene assay was performed to confirm the interaction between MALAT1 and miR-9 in chondrocytes. Following co-transfection with miR-9 mimic, the dual-luciferase reporter gene assay revealed that miR-9 significantly decreased the luciferase activity of wild-type MALAT1 compared with mutant MALAT1 (Fig. 5B). In addition, co-transfection with scramble control had no effect on the luciferase activity of wild-type or mutant MALAT1. To further investigate the role of miR-9 in OA, potential target genes of miR-9 were examined. Bioinformatics analysis was performed using the online microRNA database (www.mirdb. org) to identify NF- $\kappa$ B1 as a putative target gene of miR-9 (Fig. 5C). Following co-transfection with miR-9 mimic, the dual-luciferase reporter gene assay revealed that miR-9 significantly decreased the luciferase activity of wild-type

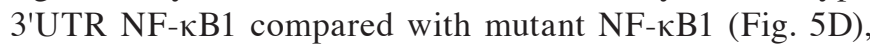
while co-transfection with scramble control had no effect on the luciferase activity of wild-type or mutant NF-kB1. Taken together, these results suggest MALAT1 directly regulated miR-9, and NF-кB1 was confirmed as a target gene of miR-9. 
A
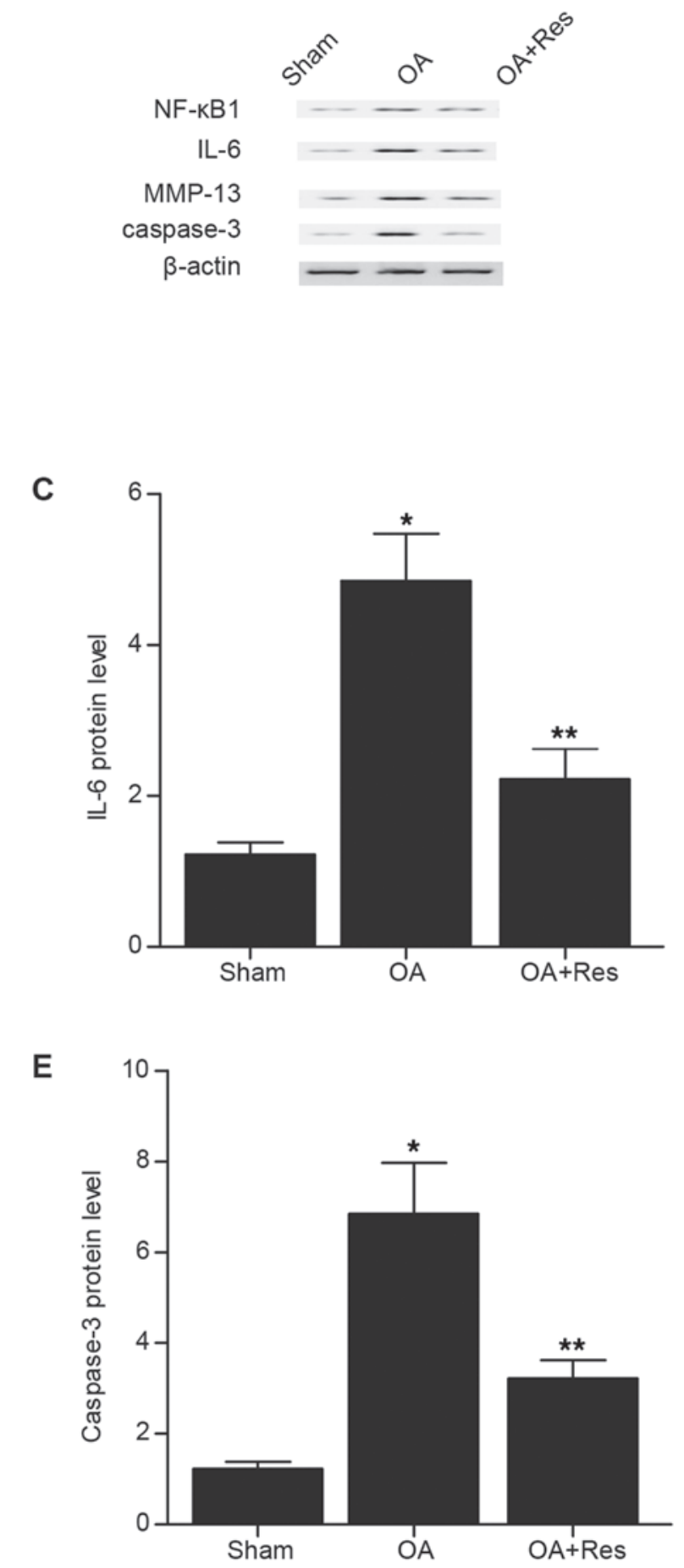

B

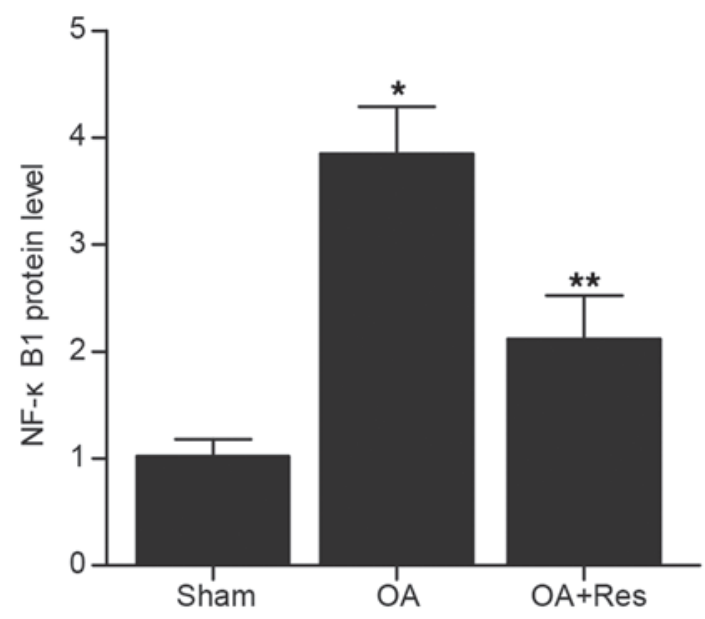

D

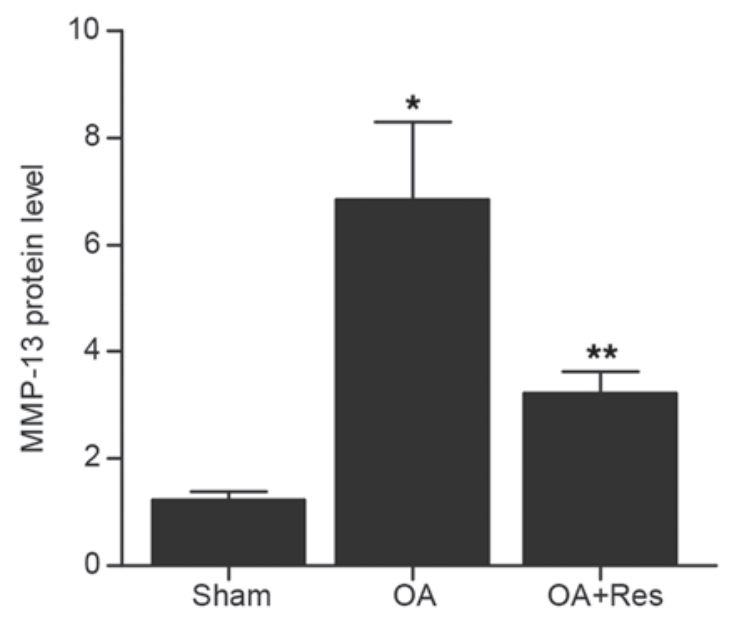

Figure 2. NF- $\kappa$ B1, IL-6, MMP-13 and caspase-3 expression in the in vivo model of OA. (A) The relative protein expression levels of NF- $\kappa$ B1, IL-6, MMP-13 and caspase- 3 were determined by western blot analysis in tissue samples from mice in the Sham, OA and OA + Res groups. Quantification of (B) NF- $\kappa$ B , (C) IL-6, (D) MMP-13 and (E) caspase-3 protein expression. ${ }^{*} \mathrm{P}<0.05$ vs. OA + Res group; ${ }^{* *} \mathrm{P}<0.05$ vs. sham group. NF- $\kappa \mathrm{B} 1$, nuclear factor kappa B subunit 1 ; IL-6, interleukin-6; MMP-13, matrix metallopeptidase 13; OA, osteoarthritis; Res, Resveratrol.

Res influences the transcriptional activity of the MALAT1 promoter. To further explore the underlying mechanism of MALAT1 in OA, a dual-luciferase reporter construct driven by MALAT1 promoter was examined in chondrocytes following treatment with Res. The luciferase reporter gene assay revealed that the transcriptional activation of the MALAT1 promoter was significantly decreased following treatment with Res in a dose-dependent manner compared with control (Fig. 6A). In addition, the relative mRNA expression level of MALAT1 significantly decreased following treatment with Res in a dose-dependent manner compared with control (Fig. 6B). Taken together, these results suggest that treatment with Res suppressed the transcriptional activity of the MALAT1 promoter thereby inhibiting MALAT1 expression. 

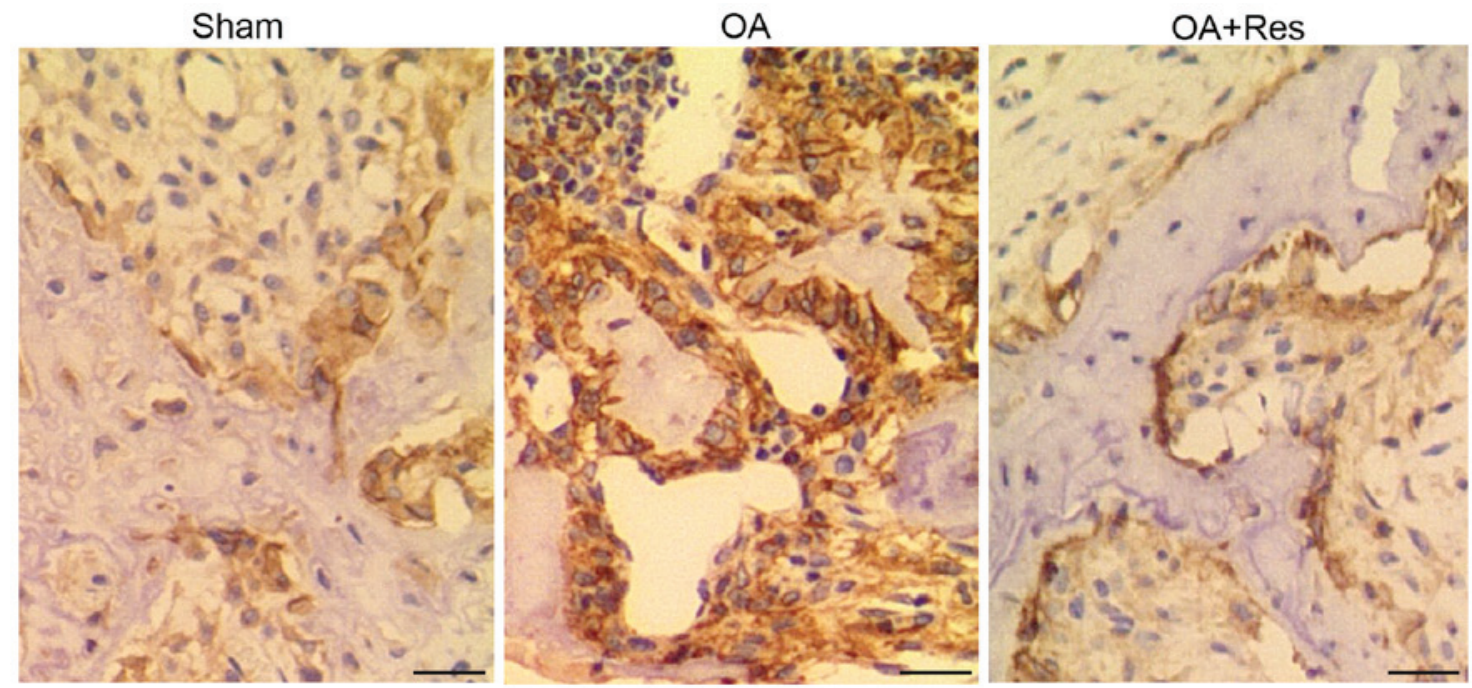

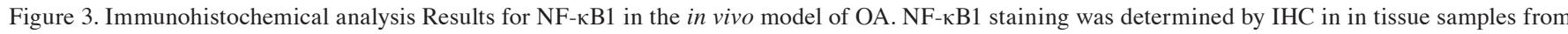

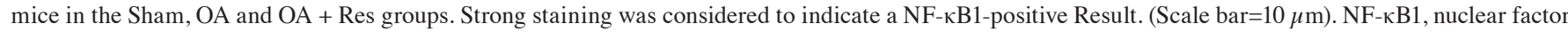
kappa B subunit 1; OA, osteoarthritis; Res, Resveratrol; IHC, immunohistochemistry.
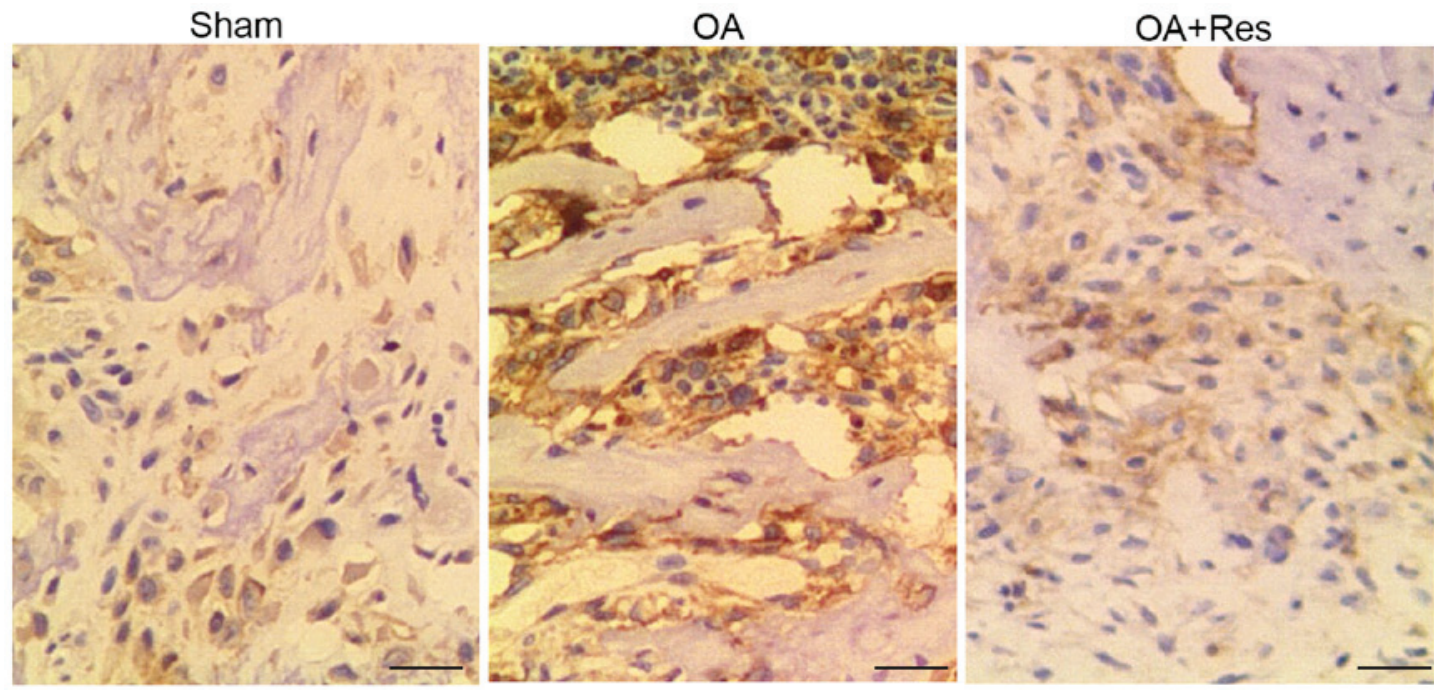

Figure 4. Immunohistochemical analysis results for MMP-13 in the in vivo model of OA. MMP-13 staining was determined by IHC in in tissue samples from mice in the Sham, OA and OA + Res groups. Strong staining was considered to indicate a MMP-13-positive Result. (Scale bar=10 $\mu \mathrm{m}$ ). MMP-13, matrix metallopeptidase 13; OA, osteoarthritis; Res, Resveratrol; IHC, immunohistochemistry.

Res effects miR-9 and the $N F-\kappa B 1$ signaling pathway in chondrocytes. The expression levels of miR-9, NF-кB1, IL-6, MMP-13, caspase-3 were detected in cells following treatment with different doses (15 and $30 \mu \mathrm{M})$ of Res. The relative expression level of miR-9 was significantly increased, whilst the mRNA expression level of NF- $\mathrm{kB} 1$ was significantly decreased following treatment with Res in a dose-dependent manner compared with control (Fig. 7A and B). Similarly, the protein expression levels of NF-kB1, IL-6, MMP-13 and caspase- 3 were significantly decreased following treatment with Res compared with control (Fig. 7C-G).

Res promotes cell proliferation in mouse chondrocytes. Following treatment with various doses $(15$ and $30 \mu \mathrm{M})$ of Res, cell viability was determined by MTT assay. The growth rate of chondrocytes significantly increased following treat- ment with Res in a dose-dependent manner compared with control (Fig. 8). These results suggest that treatment with Res can significantly increase chondrocyte proliferation.

\section{Discussion}

As an abundant phytoalexin found in grape skins and red wine, resveratrol $\left(3,4^{\prime}, 5\right.$-trihydroxystilbene) is a potent and selective inhibitor of NF- $\mathrm{kB}$ activation (18-20). In addition, Res inhibits cyclooxygenase 2 (COX-2) transcription and activity in human mammary epithelial cells $(21,22)$. As a polyphenolic phytoestrogen, Res can activate sirtuin 1, expression of which was demonstrated previously to be inhibited in OA. In addition, Res can promote the differentiation of OA and therefore Res may be beneficial in maintaining healthy cartilage (23). In the current study, the effect of Res on the transcriptional activity 
A

MiR9-MALATI 1199

GCGGCGGAUGAUGCCGACG

| | | | | | | | || || ||

CGCCG A ACUACGGCUGC

MiR9-MALATI 6229

GCGGCGGAUGAUGTAATGCGGGAUCACAGGUGCCGACG

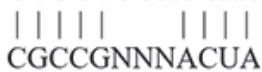

A $\quad$ G

I | | | | | |
CGGCUGC

\section{C}

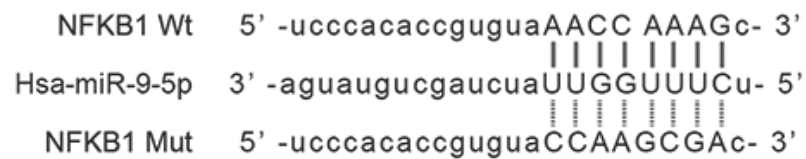

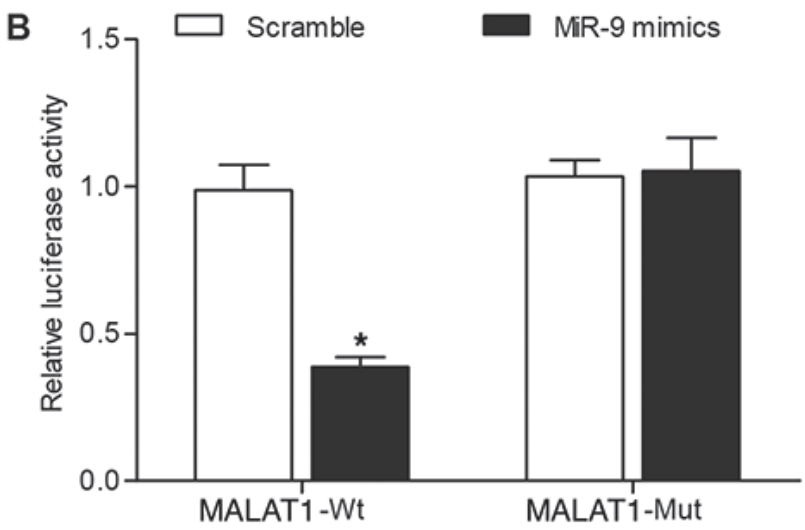

D

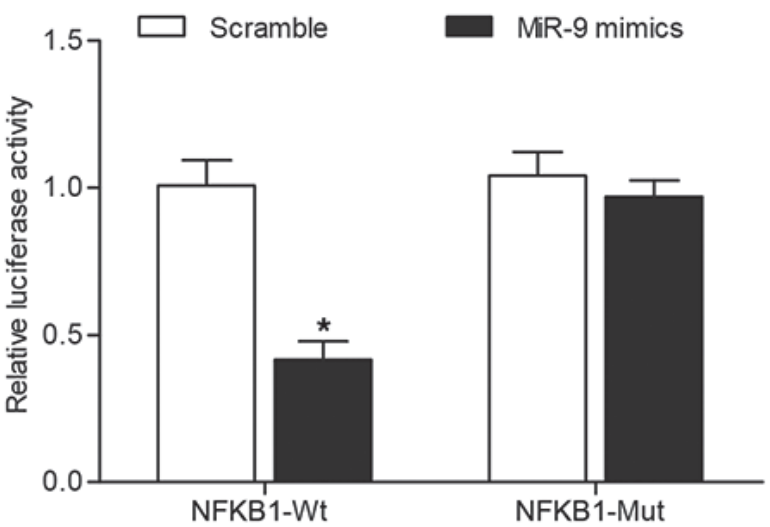

Figure 5. MALAT1 directly regulates miR-9 and miR-9 directly targets NF- $\mathrm{kB} 1$. (A) Sequence comparison between the wild-type and mutant 3'UTR of MALAT1 and miR-9. (B) Chondrocytes were co-transfected with the wild-type or mutant MALAT1 luciferase constructs and miR-9 mimic or scramble control and luciferase activities were examined following 48 -h transfection. $\mathrm{n}=3,{ }^{*} \mathrm{P}<0.05$ vs. scramble control group. (C) Sequence comparison between

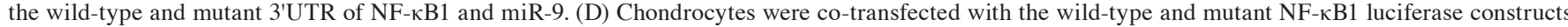
and miR-9 mimic or scramble control and luciferase activities were measured following 48 -h transfection. $n=3$, $\mathrm{P}<0.05$, vs. scramble control group. miR, microRNA; MALAT1, metastasis associated lung adenocarcinoma transcript 1; NF-кB1, nuclear factor kappa B subunit 1; UTR, untranslated region.
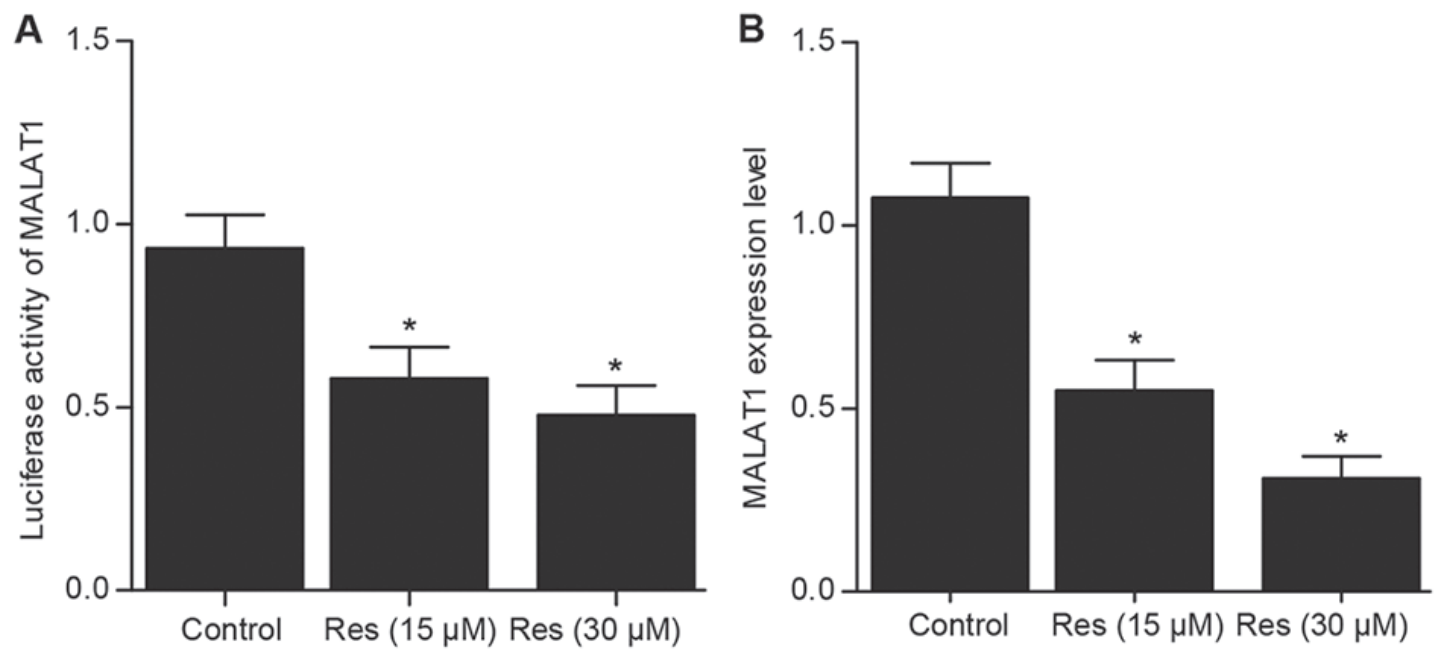

Figure 6. Treatment with Res influences the transcriptional activity of MALAT1 promoter. (A) Chondrocytes were transfected with the promoter region of wild-type MALAT1 luciferase construct and luciferase activity was examined following treatment with 0,15 or $30 \mu \mathrm{M}$ Res for $48 \mathrm{~h}$. (B) The relative MALAT1 mRNA expression level was determined by RT-qPCR in chondrocytes following treatment with 0,15 or $30 \mu \mathrm{M}$ Res for $48 \mathrm{~h}$. $\mathrm{n}=3$. ${ }^{*} \mathrm{P}<0.05 \mathrm{vs}$. control group. MALAT1, metastasis associated lung adenocarcinoma transcript 1; Res, Resveratrol; RT-qPCR, reverse transcription-quantitative polymerase chain reaction.

of the MALAT1 promoter was examined. Treatment with Res suppressed the transcriptional activity of the MALAT1 promoter thereby inhibited MALAT1 expression. In addition, RT-qPCR and western blot analysis were used to determine the relative expression levels of MALAT1, miR-9, NF-кB1, IL-6, MMP-13 and caspase- 3 in vitro and in the in vivo model of OA, as well as examining the effect of Res. Following treatment with Res, the relative expression level of miR-9 was 

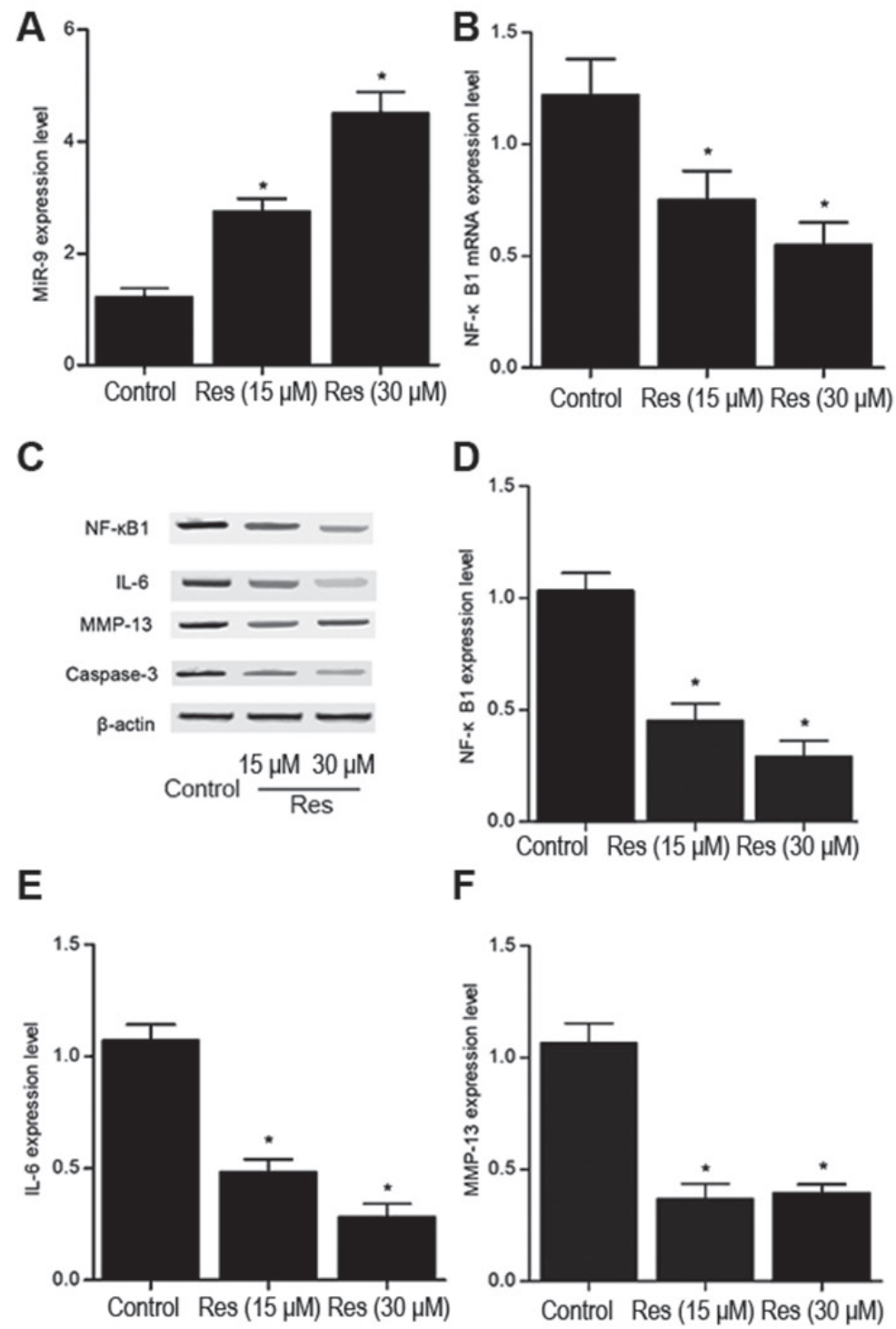

F
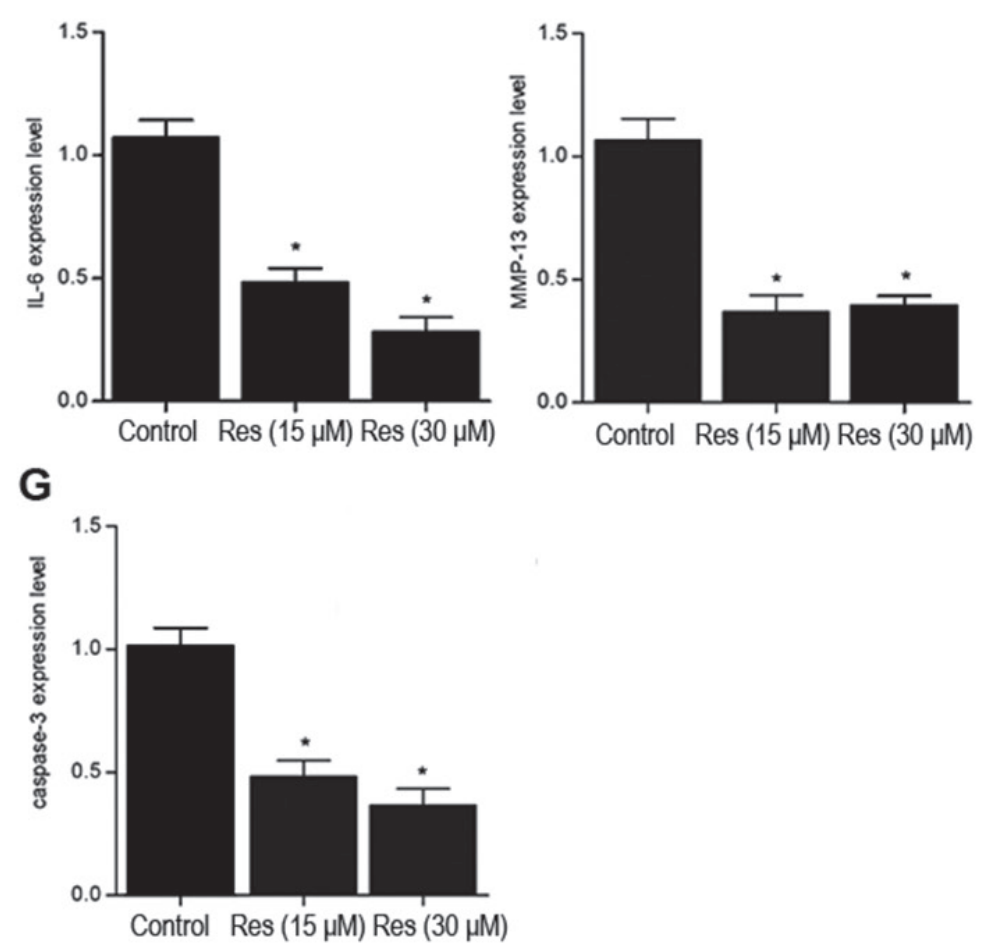

Figure 7. Effect of Res treatment on miR-9, NF-кB1, IL-6, MMP-13, caspase-3 expression in mouse chondrocytes. The relative (A) miR-9 and (B) NF- $\mathrm{kB} 1$ mRNA expression levels were determined by RT-qPCR in chondrocytes following treatment with 0,15 or $30 \mathrm{mM}$ Res for $48 \mathrm{~h}$. (C) The relative NF-kB1, IL-6, MMP-13 and caspase-3 protein expression levels were determined by western blot analysis in chondrocytes following treatment with 0,15 or $30 \mathrm{mM}$ Res for 48 h. Quantification of (D) NF-kB1, (E) IL-6, (F) MMP-13 and (G) caspase-3 protein expression. $n=3$. ${ }^{*} \mathrm{P}<0.05$ vs. control group. miR, microRNA; NF- $k B 1$, nuclear factor kappa B subunit 1; IL-6, interleukin-6; MMP-13, matrix metallopeptidase 13; Res, Resveratrol.

significantly increased in a dose-dependent manner, whereas the protein expression levels of NF-кB1, IL-6, MMP-13 and caspase-3 were significantly decreased.

A previous study demonstrated that Res reduced the expression of MALAT1 (16). To investigate the effect of Res on MALAT1 expression in OA, a dual luciferase reporter construct driven by MALAT1 promoter was examined in chondrocytes following treatment with Res. The luciferase reporter gene assay revealed that treatment with Res significantly suppressed the transcriptional activity of the MALAT1 promoter. These results suggest that Res may directly influence the transcription of MALAT1. In the current study, the 


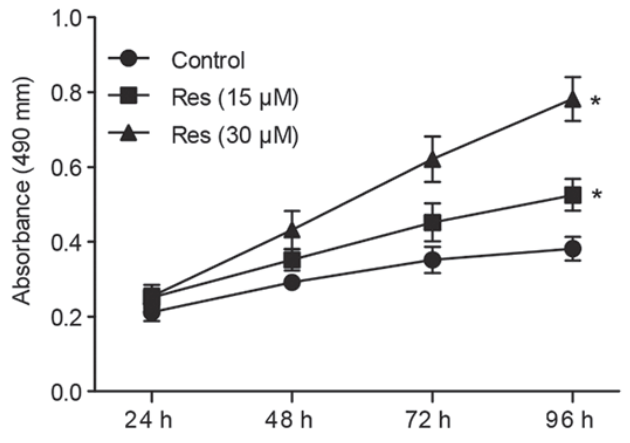

Figure 8. Res treatment increases cell proliferation in mouse chondrocytes. Cell proliferation was examined in chondrocytes following treatment with 0 , 15 or $30 \mu \mathrm{M}$ Res for 48 h. $\mathrm{n}=3$. ${ }^{*} \mathrm{P}<0.05$ vs. control group.

luciferase reporter gene assay was also used to investigate the regulatory relationship between miR-9 and MALAT1 or $\mathrm{NF}-\kappa \mathrm{B} 1$. MALAT1 directly regulated miR-9, and NF- $\kappa \mathrm{B} 1$ was confirmed as a target gene of miR-9.

Several studies have demonstrated the effect of Res on $\mathrm{NF}-\kappa \mathrm{B}(24-26) . \mathrm{NF}-\kappa \mathrm{B}$ is a transcription factor that mediates immune and inflammatory responses, and it is hypothesized that Res exerts its effects by partially inhibiting NF- $\kappa \mathrm{B}$ activation (24). Res reduces $I_{\kappa} \mathrm{B} \alpha$ degradation and prevents the nuclear translocation of $\mathrm{p} 65 \mathrm{NF}-\kappa \mathrm{B}$ in mast cells stimulated by PMA and A23187. It is likely that Res inhibits the expression of COX-2, TNF- $\alpha$, IL- 6 and IL- 8 through NF- $\kappa$ B activation and $\mathrm{I} \kappa \mathrm{B}$ degradation (24). NF- $\kappa \mathrm{B} 1$ regulates the transcription of genes which function in cell adhesion, proliferation and differentiation as well as the immune response, apoptosis and angiogenesis (25). NF- $\kappa \mathrm{B} 1$ encodes both p50 and p105 proteins (26). p105 is a cytoplasmic protein that does not bind to DNA directly. However, p50 can directly bind to DNA and is subunit is derived from the $\mathrm{N}$-terminus of the precursor protein, p105 (26). In the current study, DMM was used to establish an in vivo model of OA, and Res was used to treat OA. The summed score and the relative MALAT1, miR- 9 and NF- $\kappa$ B1 expression levels were examined in tissue samples from mice in the sham, OA and OA + Res groups. The relative mRNA expression levels of MALAT1 and NF- $\kappa \mathrm{B} 1$ were significantly increased, while miR-9 expression was significantly decreased in the OA group compared with the sham group. However, treatment with Res partially reversed the effects of OA on the mRNA expression levels of MALAT1, miR-9 and NF- $\mathrm{B} 1$. In addition, western blot analysis demonstrated that the relative protein expression levels of NF- $\mathrm{BB} 1$, IL-6, MMP-13 and caspase-3 were significantly increased in the OA and OA + Res groups compared with the sham group although treatment with Res partially reversed the effects of OA on protein expression. Furthermore, NF- $\mathrm{KB} 1$ and MMP-13 expression was examined by IHC in samples from mice in the sham, OA, $\mathrm{OA}+\mathrm{Res}$ groups. NF- $\mathrm{NB} 1$ and MMP-13 were highly expressed in the $\mathrm{OA}$ and $\mathrm{OA}+$ Res groups compared with sham group, however treatment with Res partially reversed the effects of $\mathrm{OA}$ on $\mathrm{NF}-\kappa \mathrm{B} 1$ and MMP-13 expression.

The interactions between miR-9 and NF- $\kappa \mathrm{B}$ were hypothesized to suppress apoptosis during chondrogenesis (27), as cells transfected with miR-9 inhibitors were associated with a higher level of caspase-3 activity and an increased rate of apoptosis, whereas cells transfected with miR-9 mimics were associated with a lower level of caspase-3 activity and a decreased rate of apoptosis (28). Different cell types and distinct intercellular environments may cause differences observed. In particular, a previous study reported low miR-9 expression in the knee tissues of patients with OA which may be related with the enhanced level of apoptosis in chondrocytes (27). In addition, miR-9 can negatively regulate the expression of NFkB1, thus suggesting that a reduction in miR-9 expression may increase the expression of $\mathrm{NF}-\kappa \mathrm{B}$ and therefore inhibit cell proliferation $(29,30-32)$

In conclusion, treatment with Res downregulates MALAT1 expression, and MALAT1 may function as a molecular sponge of miR-9. In addition, miR-9 can directly target NF- $\kappa$ B, and as an inflammatory cytokine $\mathrm{NF}-\kappa \mathrm{B}$ can induce apoptosis in chondrocytes contributing to the development of OA. In the current study, DMM was used to establish an in vivo model of OA, which was treated with Res and the effect of Res was examined in vitro and in the in vivo model of $\mathrm{OA}$.

\section{Acknowledgements}

Not applicable.

\section{Funding}

No funding was received.

\section{Availability of data and materials}

The data that support the findings of the present study are available from the corresponding author upon reasonable request.

\section{Authors' contributions}

GZ and ZG planned the study, HZ and WY collected the literature, GZ, HZ, WY, and ZG collected the data, XT, XL and ZG analyzed the data, GZ and ZG prepared the manuscript and all the other co-authors approved the final manuscript.

\section{Ethics approval and consent to participate}

The current study was approved by the Institutional Ethics Committee on Animal Research at Nanjing University of Chinese Medicine.

\section{Patient consent for publication}

Not applicable.

\section{Competing interests}

The authors declare that they have no competing interests.

\section{References}

1. Sasaki H, Takayama K, Matsushita T, Ishida K, Kubo S, Matsumoto T, Fujita N, Oka S, Kurosaka M and Kuroda R: Autophagy modulates osteoarthritis-related gene expression in human chondrocytes. Arthritis Rheum 64: 1920-1928, 2012. 
2. Goggs R, Carter SD, Schulze-Tanzil G, Shakibaei M and Mobasheri A: Apoptosis and the loss of chondrocyte survival signals contribute to articular cartilage degradation in osteoarthritis. Vet J 166: 140-158, 2003.

3. Csaki C, Keshishzadeh N, Fischer K and Shakibaei M: Regulation of inflammation signalling by resveratrol in human chondrocytes in vitro. Biochem Pharmacol 75: 677-687, 2008.

4. Csaki C, Mobasheri A and Shakibaei M: Synergistic chondroprotective effects of curcumin and resveratrol in human articular chondrocytes: Inhibition of IL-1beta-induced NF-kappaB-mediated inflammation and apoptosis. Arthritis Res Ther 11: R165, 2009.

5. Shakibaei M, Csaki C, Nebrich S and Mobasheri A: Resveratrol suppresses interleukin-1beta-induced inflammatory signaling and apoptosis in human articular chondrocytes: Potential for use as a novel nutraceutical for the treatment of osteoarthritis. Biochem Pharmacol 76: 1426-1439, 2008.

6. Fatica A and Bozzoni I: Long non-coding RNAs: New players in cell differentiation and development. Nat Rev Genet 15: 7-21, 2014.

7. St Laurent G, Wahlestedt C and Kapranov P: The Landscape of long noncoding RNA classification. Trends Genet 31: 239-251, 2015.

8. Mercer TR, Dinger ME and Mattick JS: Long non-coding RNAs: Insights into functions. Nat Rev Genet 10: 155-159, 2009.

9. Ponting CP, Oliver PL and Reik W: Evolution and functions of long noncoding RNAs. Cell 136: 629-641, 2009.

10. Ji P, Diederichs S, Wang W, Boing S, Metzger R, Schneider PM, Tidow N, Brandt B, Buerger H, Bulk E, et al: MALAT-1, a nove noncoding RNA, and thymosin beta4 predict metastasis and survival in early-stage non-small cell lung cancer. Oncogene 22 : 8031-8041, 2003

11. Holoch D and Moazed D: RNA-mediated epigenetic regulation of gene expression. Nat Rev Genet 16: 71-84, 2015.

12. Yoon JH, Abdelmohsen K and Gorospe M: Posttranscriptional gene regulation by long noncoding RNA. J Mol Biol 425: 3723-3730, 2013

13. Xing D, Liang JQ, Li Y, Lu J, Jia HB, Xu LY and Ma XL: Identification of long noncoding RNA associated with osteoarthritis in humans. Orthop Surg 6: 288-293, 2014.

14. Mattick JS and Makunin IV: Small regulatory RNAs in mammals. Hum Mol Genet 1: R121-R132, 2005.

15. Rodriguez A, Griffiths-Jones S, Ashurst JL and Bradley A: Identification of mammalian microRNA host genes and transcription units. Genome Res 14: 1902-1910, 2004.

16. Devor EJ: Primate microRNAs miR-220 and miR-492 lie within processed pseudogenes. J Hered 97: 186-190, 2006.

17. Ji Q, Liu X, Fu X, Zhang L, Sui H, Zhou L, Sun J, Cai J, Qin J, Ren J and Li Q: Resveratrol inhibits invasion and metastasis of colorectal cancer cells via MALAT1 mediated Wnt $/ \beta$-catenin signal pathway. PLoS One 8: e78700, 2013.

18. Gu R, Liu N, Luo S, Huang W, Zha Z and Yang J: MicroRNA-9 regulates the development of knee osteoarthritis through the NF-kappaB1 pathway in chondrocytes. Medicine (Baltimore) 95: e4315, 2016.

19. Xu L, Polur I, Servais JM, Hsieh S, Lee PL, Goldring MB and $\mathrm{Li}$ Y: Intact pericellular matrix of articular cartilage is required for unactivated discoidin domain receptor 2 in the mouse model. Am J Pathol 179: 1338-1346, 2011.

20. Livak KJ and Schmittgen TD: Analysis of relative gene expression data using real-time quantitative PCR and the 2(-Delta Delta C(T)) method. Methods 25: 402-408, 2001.
21. Manna SK, Mukhopadhyay A and Aggarwal BB: Resveratrol suppresses TNF-induced activation of nuclear transcription factors NF-kappa B, activator protein-1, and apoptosis: Potential role of reactive oxygen intermediates and lipid peroxidation. J Immunol 164: 6509-6519, 2000.

22. Estrov Z, Shishodia S, Faderl S, Harris D, Van Q, Kantarjian HM, Talpaz M and Aggarwal BB: Resveratrol blocks interleukin-1beta-induced activation of the nuclear transcription factor NF-kappaB, inhibits proliferation, causes S-phase arrest, and induces apoptosis of acute myeloid leukemia cells. Blood 102: 987-995, 2003.

23. Holmes-McNary M and Baldwin AS Jr: Chemopreventive properties of trans-resveratrol are associated with inhibition of activation of the IkappaB kinase. Cancer Res 60: 3477-3483, 2000.

24. Subbaramaiah K, Chung WJ, Michaluart P, Telang N, Tanabe T, Inoue $\mathrm{H}$, Jang $\mathrm{M}$, Pezzuto JM and Dannenberg AJ: Resveratrol inhibits cyclooxygenase-2 transcription and activity in phorbol ester-treated human mammary epithelial cells. J Biol Chem 273: 21875-21882, 1998 .

25. Surh YJ, Chun KS, Cha HH, Han SS, Keum YS, Park KK and Lee SS: Molecular mechanisms underlying chemopreventive activities of anti-inflammatory phytochemicals: Down-regulation of COX-2 and iNOS through suppression of NF-kappa B activation. Mutat Res 480-481: 243-268, 2001.

26. Shan T, Wang Y, Wu T, Liu C, Guo J, Zhang Y, Liu J and Xu Z: Porcine sirtuin 1 gene clone, expression pattern, and regulation by resveratrol. J Anim Sci 87: 895-904, 2009.

27. Salemi M, Barone C, Romano C, Scillato F, Ragalmuto A, Caniglia S, Salluzzo MG, Sciuto G, Ridolfo F and Bosco P: NF- $\kappa \mathrm{B} 1$ gene expression in Down syndrome patients. Neurol Sci 36: 1065-1066, 2015.

28. Song J, Kim D, Chun CH and Jin EJ: MicroRNA-9 regulates survival of chondroblasts and cartilage integrity by targeting protogenin. Cell Commun Signal 11: 66, 2013.

29. Tomek M, Akiyama T and Dass CR: Role of Bcl-2 in tumour cell survival and implications for pharmacotherapy. J Pharm Pharmacol 64: 1695-1702, 2012.

30. Liu N, Sun Q, Chen J, Li J, Zeng Y, Zhai S, Li P, Wang B and Wang X: MicroRNA-9 suppresses uveal melanoma cell migration and invasion through the NF- $\kappa \mathrm{B} 1$ pathway. Oncol Rep 28: 961-968, 2012.

31. Guo LM, Pu Y, Han Z, Liu T, Li YX, Liu M, Li X and Tang H: MicroRNA-9 inhibits ovarian cancer cell growth through regulation of NF-kappaB1. FEBS J 276: 5537-5546, 2009.

32. Baltaci SB, Mogulkoc R and Baltaci AK: Resveratrol and exercise. Biomed Rep 5: 525-530, 2016.

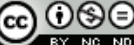

This work is licensed under a Creative Commons Attribution-NonCommercial-NoDerivatives 4.0 International (CC BY-NC-ND 4.0) License. 